\title{
Vacuum Electron Acceleration by an Intense Laser
}

\author{
P. X. Wang ${ }^{1}$, Y. K. Ho ${ }^{1 *}$, X. Q. Yuan ${ }^{1}$, Q. Kong ${ }^{1}$, \\ A. M. Sessler ${ }^{2}$, and E. Esarey ${ }^{2}$ \\ ${ }^{1}$ Institute of Modern Physics, Fudan University, Shanghai 200433, China \\ ${ }^{2}$ Ernest Orlando Lawrence Berkeley National Laboratory, University of California, \\ Berkeley CA 94720, USA \\ *To whom correspondence should be addressed. E-mail: hoyk@fudan.ac.cn
}

\begin{abstract}
Using 3D test particle simulations, the characteristics and essential conditions under which an electron, in a vacuum laser beam, can undergo a capture and acceleration scenario (CAS). When $a_{0} \gtrsim 100$ the electron can be captured and violently accelerated to energies $\gtrsim 1 \mathrm{GeV}$, with an acceleration gradient $\gtrsim 10 \mathrm{GeV} / \mathrm{cm}$, where $a_{0}=e E_{0} / m_{e} \omega c$ is the normalized laser field amplitude. The physical mechanism behind the CAS is that diffraction of the focused laser beam leads to a slowing down of the effective wave phase velocity along the captured electron trajectory, such that the electron can be trapped in the acceleration phase of the wave for a longer time and thus gain significant energy from the field.
\end{abstract}

PACS number(s): 41.75.Jv, 42.50.Vk, 42.65.Sf 
The rapid development of intense laser technology in recent years has stimulated many frontier research areas in both applied and fundamental physics [1,2]. Among them, the far-field laser acceleration of free electrons in vacuum has received wide attention [3]. Along this line, we study a laser-electron interaction model with a realistic laser beam and electrons injected upon the laser beam. The electron energy change due to the interaction is investigated by utilizing a 3D-computer simulation code to solve the relativistic Newton-Lorentz equation of motion [4]. As previously noted in simulations, although not fully understood, the most surprising and meaningful result is that when $a_{0} \gtrsim 100$, the electron can be captured and violently accelerated to energies $\gtrsim 1 \mathrm{GeV}$, with an acceleration gradient $\gtrsim 10 \mathrm{GeV} / \mathrm{cm}$, where $a_{0}=e E_{0} / m_{e} \omega c$ is a dimensionless parameter specifying the magnitude of the field, $-e, m_{e}$ are the electron charge and mass, respectively, $c$ is the speed of light in vacuum, and $\omega$ is the angular frequency of the electromagnetic wave [4,5]. We call this process CAS (Capture \& Acceleration Scenario). In the CAS regime, the energy gain is found to scale as $a_{0}^{2}$. In this Letter, a physical understanding and a comprehensive numerical study of the CAS process is presented.

The numerical simulation methods used here are similar to those we have used previously $[4,5]$. The laser beam we adopted is the lowest-order Hermite-Gaussian $(0,0)$ mode with analytical expressions in the paraxial approximation, and is polarized in the $\mathrm{x}$-direction and propagates along the $\mathrm{z}$-axis. It should be pointed out that the results given in this paper are found to be correct even if the high-order corrections to the paraxial expressions for continuous Gaussian beams [6,7] are taken into account.

For the case of a laser with a finite pulse duration $\tau$, the paraxial field components can be multiplied by a time envelope profile $f(c t-z)$, assumed to be Gaussian [8] 


$$
f(c t-z)=\exp \left(-\frac{(t-z / c)^{2}}{\tau^{2}}\right)
$$

where additional corrections to the fields of order $1 / \omega \tau$ have been neglected. Obviously, $\tau \rightarrow \infty$ in the time envelope factor $f(c t-z)$ corresponds to the case of continuous laser beams. Esarey et al. [9] have derived solutions to the wave equation valid for laser pulses of ultra-short duration. They find, for example, that the field envelope travels with a group velocity $v_{g}$, i.e., $f\left(v_{g} t-z\right)$, where $v_{g} / c=1-2 / k^{2} w_{0}^{2}$ near the laser focus, $k=\omega / c$, and $w_{0}$ is the laser spot size at focus. This effect can be neglected, however, provided that the envelope slippage $\Delta L=z\left(1-v_{g} / c\right)$ is small compared to the pulse length $L=c \tau$ over the interaction distance $z$. This implies $k L>>z / Z_{R}$ (or $k L>>1$ since $z \approx Z_{R}$ ), where $Z_{R}=k w_{0}^{2} / 2$ is the Rayleigh length.

We use a four-dimensional energy-momentum configuration to specify the electron state $\left(\gamma, P_{x}, P_{y}, P_{z}\right)$, where the Lorentz factor $\gamma$ and the momentum $P_{i}$ are normalized in the units of $m_{e} c^{2}$ and $m_{e} c$, respectively. Also, throughout the paper, time and length are normalized by $1 / \omega$ and $1 / k, \phi_{0}$ is the wave initial phase, $\theta=\tan ^{-1}\left(P_{x i} / P_{z i}\right)$ is the electron injection angle with respect to the $\mathrm{z}$-axis and $b_{0}$ the impact parameter. The electron dynamics are governed by the following relativistic Newton-Lorentz equations.

$$
\begin{aligned}
& \frac{d \mathbf{P}}{d t}=-e(\mathbf{E}+\mathbf{V} \times \mathbf{B}), \\
& \mathbf{P}=\boldsymbol{N}, \quad \gamma=\frac{1}{\sqrt{1-\mathbf{V}^{2}}},
\end{aligned}
$$

where $\mathbf{V}$ is the electron velocity in the unit of $c$. Without losing generality, we assume that the pulsed beam center reaches the point $x=y=z=0$ at $t=0$, and $\Delta t_{d}$ specifies the relative delay between the laser pulse and the electron [6]. The incoming and outgoing energy and momentum of the electron are denoted by $\left(\gamma_{i}, P_{x i}, P_{y i}, P_{z i}\right)$ and $\left(\gamma_{f}, P_{x f}, P_{y f}, P_{z f}\right)$, respectively.

Our study shows that the CAS electron dynamic regime emerges only when the laser intensity is strong enough, $a_{0} \gtrsim 100$, and when the electron injection angle 
$\theta=\tan ^{-1}\left(P_{x i} / P_{z i}\right)$ is sufficiently small. The phase space of the electron incoming momenta required by CAS is not small and readily achievable in experiments. Especially, the optimum incident momentum is not very sensitive to the laser intensity, and can be in the range 12.5-17.5 MeV. Fig.1 shows two typical cases of electron dynamics, i. e., CAS trajectory and the electron inelastic scattering (IS). A stationary laser field (continuous laser beam) with intensity $a_{0}=100$ was used. Other parameters are $w_{0}=150, P_{x i}=3.5, P_{y i}=0, P_{z i}=35, b_{0}=0$. Fig.1(a) shows electron trajectories in the $x$ $z$ plane. It clearly indicates whether the electron is captured or scattered by the laser beam. In Fig.1(b), we present the electron energy $\gamma$ as a function of time. Fig.1(c) shows the variation of the laser phase $\varphi$ experienced by an electron during the interaction. For the CAS, the electrons can be captured into the intense field region rather than expelled from it and the captured electrons can be accelerated to $\mathrm{GeV}$ energies with acceleration gradients of tens of $\mathrm{GeV} / \mathrm{cm}$.

Fig.2 shows that the electron outgoing energy $\gamma_{f}$ as a function of the relative delay time $\Delta t_{d}$. Without losing generality, we chose $\phi_{0}=0, \tau=500$ and other parameters are the same as those in Fig.1. Fig.2 corresponds to the case of an incident electron bunch, but only those electrons moving in the $x-z$ plane $\left(b_{0}=0\right)$ in the bunch are taken into account. Obviously, the output of the acceleration mechanism is a $\mathrm{GeV}$ electron macropulse that consists of many micro-pulses. Each of the micro-pulses has the same shapefactor which can be seen from the insets of Fig.2. The macro-pulse corresponds to the duration of the laser pulse, and the micro-pulse to the periodicity of the laser wave. Those output features are analogous to that of conventional linacs.

For a realistic incident electron bunch with a certain width (spot size), we have to study the dependence of the acceleration effect on the impact parameter $b_{0}$. Fig.3 shows that the electron maximum outgoing energy $\gamma_{f \max }\left(\gamma_{f \max }\right.$ is the maximum $\gamma_{f}$ in the whole phase range $\phi_{0} \in[0,2 \pi]$ as functions of the impact parameter $b_{0}$ and the relative delay time $\Delta t_{d}$. Other parameters are the same as those in Fig.2. From Fig.3, we see that the output electron bunch has a large (100\%) energy spread with energies in excess of a $\mathrm{GeV}$. Furthermore, the bunch can have a physical size comparable to that of the laser pulse. Provided the incident electron bunch is formed with a size comparable to, 
or less than, that of the laser pulse, the total fraction of electrons captured and accelerated to $\mathrm{GeV}$ energy by the CAS mechanism can reach more than $20 \%$. Thus we see that the CAS accelerator mechanism is fairly efficient. Combined with an extra-high acceleration gradient and stable output, the CAS mechanism is an attractive alternate to plasma-based laser acceleration schemes [2].

Although the energy gain in the CAS regime scales as $a_{0}^{2}$, it cannot be described by a time-averaged ponderomotive model [10] or by a simple linear acceleration model [11]. Simulations [12] comparing the solution of a time-averaged ponderomotive model to the full (not time averaged) Lorentz equations of motion indicate that the ponderomotive model is fairly accurate in the regime $a_{0}<10$. However, in the CAS regime $\left(a_{0}>50\right)$, the ponderomotive model is found to be highly inaccurate and predicts low energy gains $(<50 \mathrm{MeV})$.

To explain the mechanism leading to large energy gains in CAS, it is instructive to observe the phase variation experienced by the electron in the wave field. The most prominent feature of Fig.1(c) is that the phase experienced by the CAS electron varies extremely slowly even in the early acceleration stage. As we know, the phase slippage velocity of an electron in a vacuum electromagnetic plane wave field can be approximately estimated by $\omega /\left(2 c \gamma_{\| /}^{2}\right)$, where $\gamma_{/ /}=1 / \sqrt{1-V_{/ /}^{2}}$ and $V_{/ /}$is the velocity along the wave propagation direction. Thus it would be expected that when $\gamma_{/ /}$is not large, as in the early acceleration stage, there should be noticeable phase slippage. To study the physical basis of this phenomenon, we note that the laser field concerned is not a plane wave, but a Gaussian beam field where the radius of the curvature varies due to the diffraction effect of the optical beam. The phase of a Gaussian beam field is [10]

$$
\varphi=k z-\omega t-\phi(z)-\phi_{0}+\frac{k\left(x^{2}+y^{2}\right)}{2 R(z)}
$$

where $\phi(z)=\tan ^{-1}\left(z / Z_{R}\right)$ is the Gouy phase shift, and the radius of the curvature $R(z)=z\left(1+Z_{R}^{2} / z^{2}\right)$ first decreases from $z=0$ to $Z_{R}$, and then increases from $Z_{R}$ to infinity. The wave phase velocity along a particle trajectory can be calculated via

$$
\frac{\partial \varphi}{\partial t}+\left(V_{\varphi}\right)_{J}(\nabla \varphi)_{J}=0
$$


where $\left(V_{\varphi}\right)_{J}$ is the phase velocity of the wave along the trajectory and $(\nabla \varphi)_{J}$ the gradient of the phase field along the trajectory.

Fig.4(a) denotes the effective phase velocity along the electron dynamic trajectory $\left(V_{\varphi}\right)_{J}$ for the cases of CAS (solid line) and IS (dotted line). Fig.4(b) and Fig.4(c) compare the wave phase velocity along the electron trajectory with the electron velocity for CAS and IS, respectively. From Fig.4(c) we can see the wave phase velocity for the IS trajectory is much faster than the electron dynamic velocity. Thus the electron phase slippage in the wave will be very fast as shown in Fig.1(c). As a consequence, the electron cannot get considerable net energy gain from the laser field. In contrast to that, from Fig4(b), we see that in the path between 0 and $Z_{R}$, the wave phase velocity of the CAS trajectory is even less than the electron velocity, and in the following path, the effective phase velocity is kept very close to the electron velocity. This is the reason that the phase slippage of the electron in the wave field remains extremely low. Consequently, the electron can be trapped in the acceleration phase for long times to gain considerable energy from the laser field.

In summary, use is made of 3D test particle simulations to study the motion of free electrons in an intense laser field. The most prominent feature of those dynamic trajectories is that the incident electron can be captured into the intense field region, rather than expelled from it as predicted by the conventional ponderomotive potential model. Violent acceleration to energies in excess of a $\mathrm{GeV}$ in few centimeters results. The output of the CAS acceleration mechanism is a relativistic electron macro-pulse (the duration of the laser pulse) composed of many micro-pulses (the periodicity of the laser wave). The physical mechanism underlying the CAS is that when an electron is captured, due to laser diffraction near the focus, the effective wave phase velocity along the dynamic trajectory of the captured particle can be less than $c$, or even less than the speed of the particle. Thus the captured electron can be kept in the acceleration phase of the wave for long times, and gain considerable energy from the laser field. It is also found that the emergence of CAS trajectories is sensitive to the laser wave phase experienced by the incident electron when it reaches the laser intense region. Provided the incident electron bunch is formed with a size comparable to, or less than, that of the laser pulse, the total fraction of electrons captured and accelerated to $\mathrm{GeV}$ energy by the CAS mechanism can reach more than $20 \%$. For some applications, the large spread in 
energies in the bunch is not acceptable and will require an electron spectrometer or tailoring the input electron beam, i.e., restricting its initial position, angle, and pulse structure.

This work is supported partly by National Natural Science Foundation of China under contract No. 19984001, National High-Tech ICP Committee in China, and Engineering-Physics Research Institute Foundation of China. A. Sessler and E. Esarey are support by the U.S. Department of Energy, Director, Office of Science, Contract No. DE-AC03-76SF00098, and acknowledge the assistance of E. Moshkovich.

\section{REFERENCES}

1. Strick, D., and Mourou, G., Opt. Commun. 56, 219 (1985); Mourou, G. A., Barty, C. P., and Perry, M. D., Phys. Today, 22, Jan. (1998); Perry, M. D., and Mourou, G. A., Science 264, 917 (1994); Blanchot, N., et al., Opt. Lett, 20, 395 (1995); Rouyer, G., et al., ibid. 13, 55 (1995).

2. Modena, Najmudln, Z., et al. Nature 377, 606 (1995); Zhang, J., et al., Science, 276, 1097 (1997); Sprangle, P., Esarey, E., Krall, J., Phys.Plasmas 3, 2183 (1996); Umstadter, D., et al., Science 273, 472 (1996); Moore, C. I., et al., Phys. Rev. Lett. 82, 1688 (1999); Ting, A., et al., Phys. Plasmas 4, 1889 (1997); Krushelnick, K., et al., Phys. Rev. Lett. 83, 737 (1999); Amiranoff, F., et al., Phys. Rev. Lett. 81, 995 (1998); Nakajima, K., et al., Phys. Rev. Lett. 74, 4428 (1995); Sprangle, P., Esarey, E., Ting, A., Joyce, G., Appl. Phys. Lett. 532146 (1988).

3. Malka, G., Lefebvre, E., and Miquel, J. L., Phys. Rev. Lett. 78, 3314 (1997); Phys. Rev. Lett. 80, 1352 (1998); McDonald, K. T., Phys. Rev. Lett. 80, 1350(1998); Moore, C. I., Knauer, J. P., and Meyerhofer, D. D., Phys. Rev. Lett. 74, 2439 (1995); Hartemann, F. V., et al., Phys. Plasmas. 6, 4104 (1999); Bucksbaum, P. H., Bashkansky, M., and McIlrath, T. J., Phys. Rev. Lett. 58, 349 (1987); Monot, P., Auguste, T., Lompré, L.A., Mainfrary, G., and Manus, C., Phys. Rev. Lett. 70, 1232 (1993); Y. I. Salamin and C. H. Keitel, Appl. Phys. Lett. 77, 1082 (2000).

4. Ho, Y. K., Wang, J. X., et al., Phys. Lett. A 220, 189 (1996); Wang, J. X., Ho, Y. K., et al., Phys. Lett. A 231, 139 (1997); Wang, J. X., Ho, Y. K., and Scheid, W., Phys. Lett. A 234, 415 (1997).

5. Zhu, L. J., Ho, Y. K., et al., Phys. Lett. A 248, 319 (1998); Wang, J. X., Ho, Y. K., et al., Phys. Rev. E 58, 6575(1998).

6. Davis, L. W., Phys. Rev. A 19, 1177 (1979).

7. Barton, J. P., and Alexander, D.R., J. Appl. Phys. 66, 2800 (1989).

8. Wang, J. X., Ho, Y. K., et al., Phys. Rev. E 60, 7473 (1999).

9. Esarey, E., et al., J. Opt. Soc. Am. B 12, 1695 (1995); Esarey, E., and Leemans, W.P., Phys. Rev. E 59, 1082 (1999). 
10. Stupakov, G.V., and Zolotorev, M.S. (submitted for publication) consider acceleration of electrons from rest to several $\mathrm{MeV}$ by the time-averaged ponderomotive laser force.

11. Woodward, P.M., J. Inst. Electr. Eng. 93, 1554 (1947); Lawson, J.D., IEEE Trans. Nucl. Sci. NS-26, 4217 (1979); Esarey, E., et al., Phys. Rev. E 52, 5443 (1995). The Lawson-Woodward theorem describes linear acceleration in which the electron moves in a straight line. In our work the electron orbits are highly nonlinear.

12. Wang, P.X., Ho, Y.K., et al., Phys. Rev. E (submitted).

13. Milonni, P. W., and Eberly, J. H., Lasers, (John Wily \& Sons, New York, 1988). 

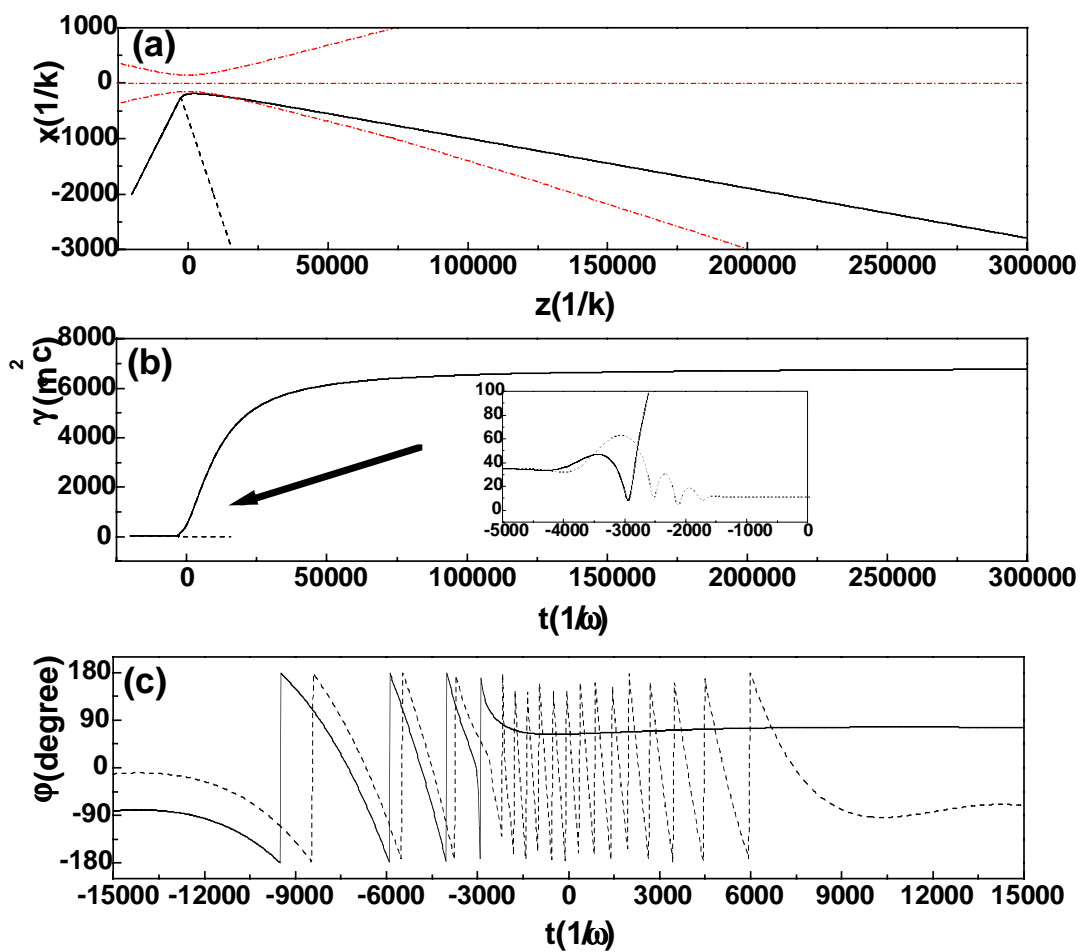

FIG. 1. Two typical cases of electron dynamics. Capture and acceleration scenario (CAS) is given by the solid line and inelastic scattering (IS) is given by the dotted line. A continuous laser beam with field intensity $a_{0}=100$ is used. Other parameters are $k w_{0}=150, k Z_{R}=11,250, P_{x i}=3.5, P_{y i}=0$, and $P_{z i}=35$. In the electron capture case, we terminate the calculation at $\omega_{t}=3 \times 10^{5}$. (a) Electron trajectories in the $x-z$ plane. The dot-dashed lines show the space profile of focused laser beam. (b) Electron energy $\gamma$ as a function of time. The inset is the enlargement of the region denoted by the arrow shown in Fig.1(b). (c) The laser wave phase experienced by the electron as a function of time. 


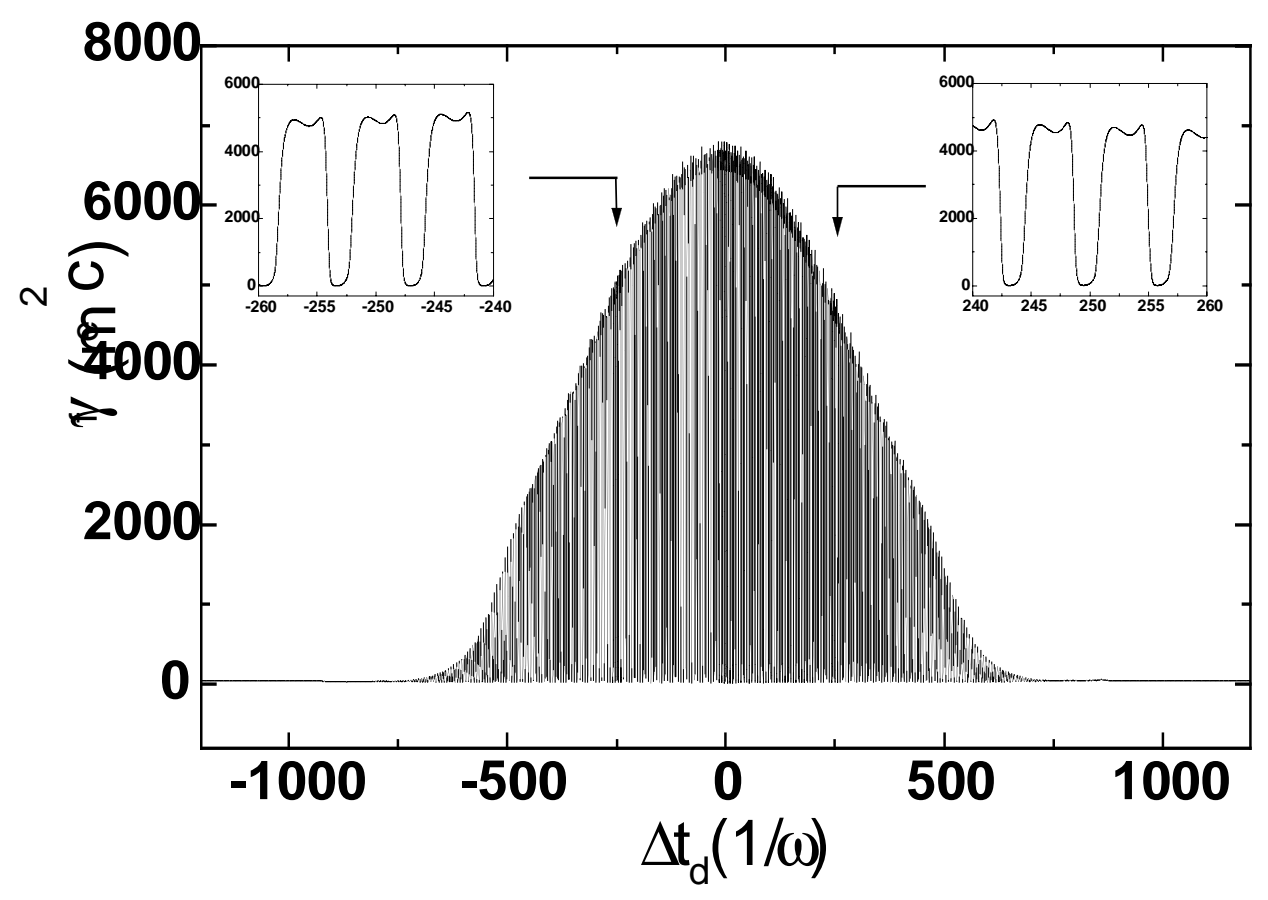

FIG. 2. Dependence of the electron final energy $\gamma_{f}$ on the relative delay time $\Delta t_{d}$ when $\phi_{0}=0$. A pulsed laser beam with $\omega \tau=500$ is used. Other parameters are the same as those in Fig.1. The insets are the enlargement of the parts denoted by the arrows shown in Fig.2. 


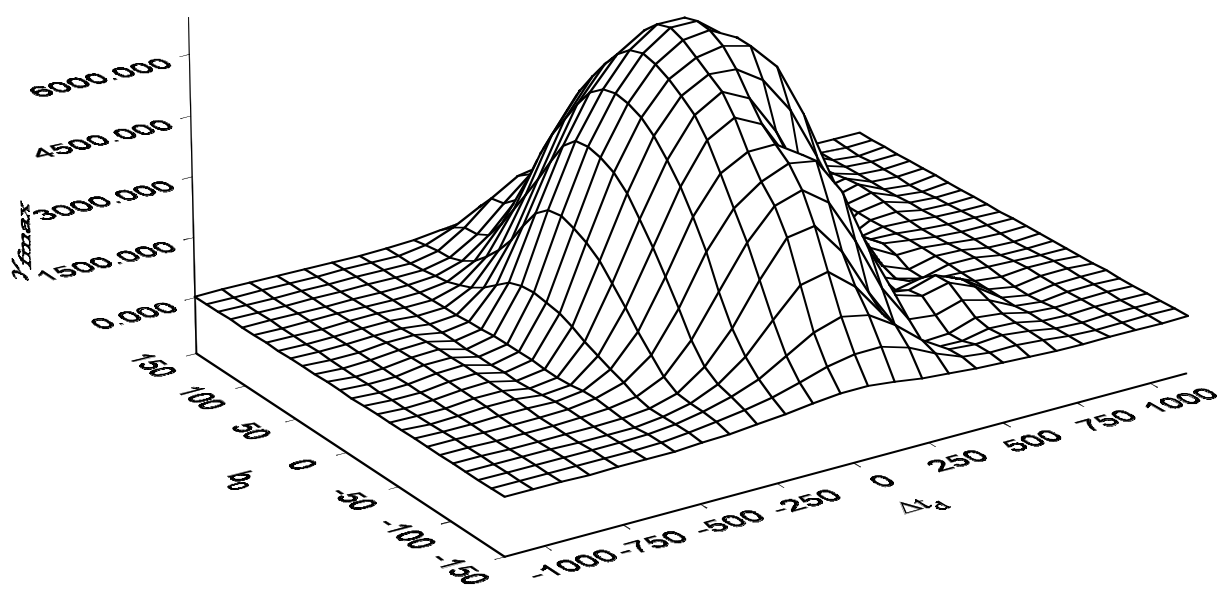

FIG. 3. The electron maximum outgoing energy $\gamma_{f \max }$ as a function of $\Delta t_{d}$ and the impact parameter $b_{0}$. Other parameters chosen are the same as those in Fig.2 


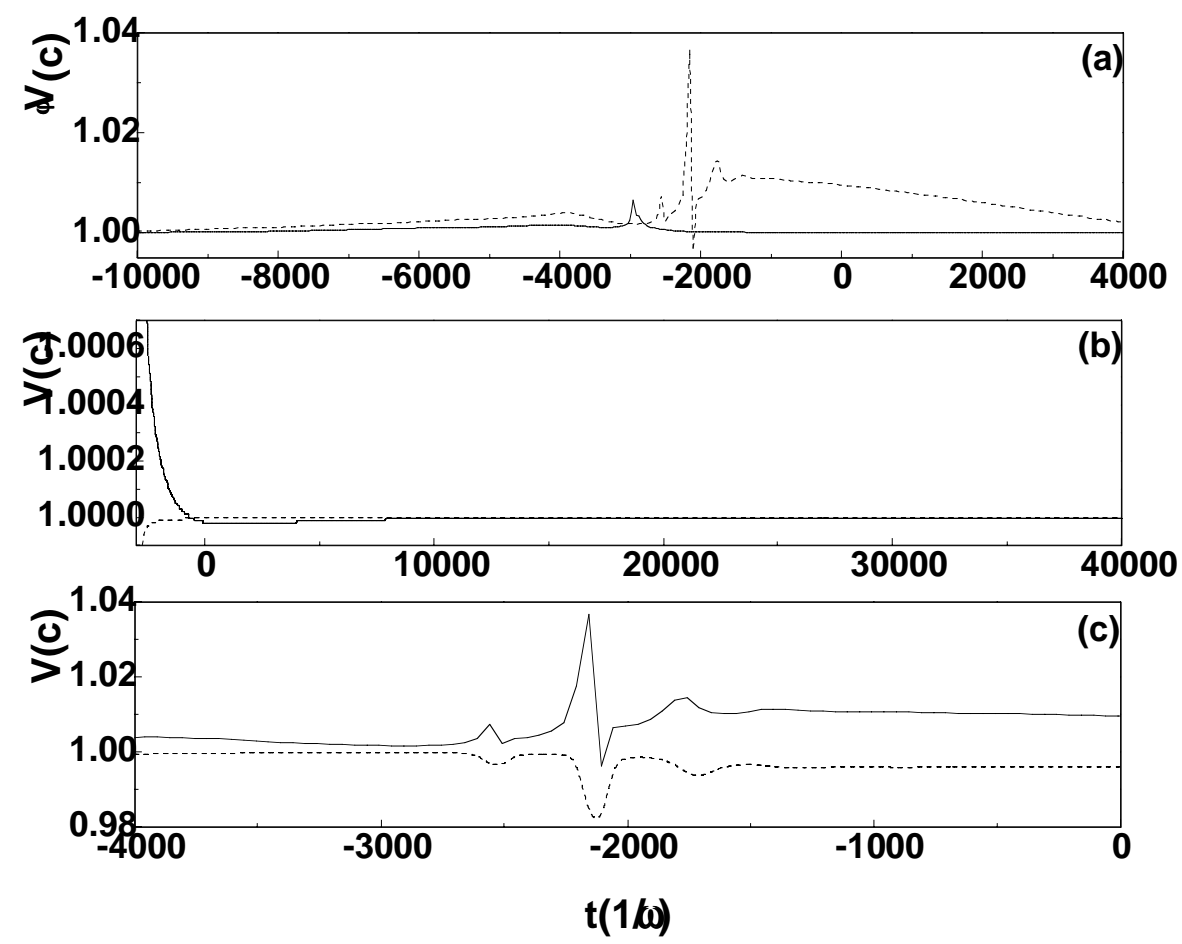

FIG. 4. (a) Variation of effective phase velocities of Gaussian laser waves along electron trajectories of CAS (solid line) and IS (dotted line) respectively. (b) Wave phase velocity along the CAS trajectory (solid line) compared with the electron's velocity (dotted line). (c) Same as Fig4(b) but for IS. The parameters chosen are the same as those in Fig.1 